\title{
Funktionale Erklärung eines südindischen Māhātmyas Die Legende Hiranyavarmans und das Leben des Cōla a-Königs Kulottunga I.
}

\author{
Von \\ HERMANN KULKE \\ Heidelberg
}

C. C. Berg, Professor für indonesische und austronesische Sprachen an der Universität Leiden, hat in den vergangenen Jahrzehnten die javanische Geschichtsschreibung einer recht kritischen Analyse unterzogen und ist dabei in bezug auf die Glaubwürdigkeit einzelner historischer Quellen zu Schlüssen gekommen, die das bisherige Bild von der frühen und mittelalterlichen Geschichte Indonesiens in wichtigen Punkten in Frage stellen. Ursache zu einigen früheren Fehleinschätzungen ist die Tatsache, daß, nach Bergs Meinung, der "optative Charakter" ${ }^{1}$ zahlreicher javanischer Texte, die er an anderer Stelle auch als "Wunschtraumdichtung ${ }^{*}$ bezeichnet ${ }^{2}$, bisher nicht hinreichend beachtet worden ist.

Den Ausgangspunkt für die kritische Beurteilung der javanischen historischen Quellen bildet für Berg der in Java besonders tief verankerte Glaube an die Macht der Wortund Schriftmagie, für den Berg in seinen zahlreichen Schriften beredte Beispiele vorträgt. In dieser Welt magischen Denkens der „alten javanischen Gesellschaft verwendete der Priester seine Macht oder die Macht des feierlich gesprochenen Wortes, um das Los seines Herrn, des Königs, damit zu lenken. Er verfaßte dazu ein Gedicht, worin er aufschrieb, wie er sich das Los seines Königs wünschte ... Das Schicksal des Königs ... wurde nicht direkt beschrieben, sondern allegorisch durch die Auswabl einer Geschichte aus der beiligen indischen Literatur, die mehr oder weniger zu der Situation paßte, worin und wofür die kultische Handlung verrichtet wurde ${ }^{*}$.

Nach Bergs Meinung ist es demnach für die Rekonstruktion der javanischen Geschichte nicht ausreichend, einen Text zu lesen und dabei die Details herauszugreifen, die hierzu geeignet erscheinen. Die Hauptfrage müsse dagegen zunächst immer wieder sein: ${ }^{\alpha} W b y$ did the man write his book, and why did be write it thus? ${ }^{4}$ Verläßt sich dennoch ein Historiker bei der Darstellung der indonesischen Geschichte auf eine wortgetreue Interpretation der vorhandenen Quellen, so muß dies die unvermeidliche Folge haben, daß das Bild, das er aus diesen Quellen rekonstruiert, genau dem Bild der Vergangenheit entspricht, „that some ancient priests have wanted us to believe in ${ }^{\alpha}{ }^{5}$, denn "a priestly doctrine is likely to invert a prevailing picture of the present ${ }^{\alpha{ }^{6}}$.

Die Ergebnisse der neuen Interpretation der javanischen Quellen durch Berg scheinen inzwischen von der westlichen Indonesien-Forschung weitgehend akzeptiert

\footnotetext{
1 Berg (1961) S. 16 - Abkürzungs- und Literaturverzeichnis am Schluß der vorliegenden Arbeit.

2 Berg (1956) S. $172 .{ }^{3}$ Ebd., S. 171. ${ }^{4}$ Berg (1961) S. $18 .{ }^{5}$ Ebd., S. 17.

- Berg (1965) S. 91.
} 
worden $\mathrm{zu} \operatorname{sein}^{7}$. Weniger Anerkennung bzw. Anwendung scheint hingegen seine Methode, die er selber als die einer „funktionalen Interpretation ${ }^{\alpha} 8$ bezeichnet, außerhalb ihres bisherigen Anwendungsbereiches, der indonesischen Geschichte, gefunden zu haben.

Im folgenden sei nun eine „funktionale Interpretation ${ }^{*}$ einer südindischen Legende vorgetragen, die im Cidambaramāhātmya $(=\mathrm{Cm})$, der Legendensammlung Cidambarams, enthalten ist, der bedeutendsten śivaitischen Tempelstadt Südindiens. Hierdurch soll aufgezeigt werden, daß Bergs Methode - wenn auch mit einer Einschränkung, auf die abschließend noch kurz eingegangen werden soll - auch auf gewisse indische Texte anwendbar ist und entscheidend zu deren Klärung beitragen kann.

Wie Cidambaram, so besitzen nahezu alle bedeutenden Wallfahrtsorte Indiens Sammlungen von Legenden und von Hymnen auf die Heiligkeit des Ortes, die unter dem Namen Māhätmya bekannt sind. Die Māhätmya stellen, wie schon ihr Name besagt", eine Verherrlichung der Macht und der Heiligkeit des Ortes dar, deren Namen sie tragen. Mit ihnen wenden sich die Priester der einzelnen heiligen Orte insbesondere an die zahlreichen Pilger, die auch heute in Indien noch von einem Wallfahrtsort zum anderen ziehen, und deren Bild jedem Indienreisenden vertraut ist. Die Māhātmyas stellen bisweilen Sammlungen verschiedener Legenden dar in denen eine bestimmte Gotheit gepriesen oder ein berühmter Wallfahrtsort gepriesen wird. Das spezifisch Eigenartige der Māhätmyas ist es aber, daß in ihnen im allgemeinen die Taten einer Gottheit im Zusammenhang mit ein e r heiligen Stätte geschildert wird. Diese Eigenart der Māhātmyas entspricht der Bhakti-Religiosität, eine bestimmte Emanation oder Inkarnation der bevorzugten Gottheit des hinduistischen Pantheons an ein em bestimmten Ort zu verehren ${ }^{10}$. Bei der auch für einen Hindu kaum zu überblickenden Vielfalt hinduistischer Götter und der unübersehbaren Vielzahl heiliger Orte in Indien versteht es sich von selber, daß dem Māhātmya eines Wallfahrtsortes eine entscheidende Rolle in der Verkündung und Propagierung der Heiligkeit dieses Ortes zufällt. Diese Verkündung geschieht meist in der Form von Legenden, bisweilen aber auch mittels philosophischmagischer Spekulationen, die die Heiligkeit eines Ortes begründen sollen ${ }^{11}$. Die folgenden Ausführungen werden einen weiteren, bisher wenig beachteten Aspekt der Māhätmyas

${ }^{7}$ Nicht dagegen z. B. von dem indonesischen Historiker und Politiker Prof. H. Muhammad Yamin: Pantjawarsa. Die Gliederung der allgemeinen und der indonesischen Geschichte in fünf Epochen (Vortrag während des Internationalen Orientalisten-Kongresses in München, 20. 8. bis 4. 9. 1957).

8 Berg (1961) S. 17.

- Das Petersburger Wörterbuch übersetzt māhātmya u. a. mit "Hohes Wesen, Größe, Majestät, Macht".

10 In Abwandlung des berühmten Terminus Max Müllers, Henotheismus, prägte der sowjetische Dravidologe Pyatigorskiy für das geschilderte Phänomen der spezifisch südindischen BhaktiReligiosität den Ausdruck „Henolocotheismus".

${ }_{11}$ Ein hervorragendes Beispiel bildet hierzu die Lehre Cidambarams, als „Pundarīkapura ${ }^{\circ}$ (ein weiterer Name Cidambarams) die Lotusstadt der Erde zu sein ( $\mathrm{Cm}, \mathrm{XV}, 18-39)$. Mikrokosmisch-makrokosmische Spekulationen über die magische Identität von menschlichem Körper und der Erde sollen auf der Grundlage der Upanișad-Lehre, daß die erlösende Erkenntnis von der Einheit Atman / Brabmans im "Lotushaus" des Herzens des menschlichen Körpers stattfindet, begründen, daß die erlösende Vereinigung mit Siva nur durch den Besuch (sevā) des Lotusherzens der Erde, nämlich Cidambaram, erreicht werden kann (siehe Kulke, Cidambaramāhātmya; Abschnitt über: Die philosophische Bedeutung Cidambarams als „Pundarika-pura ${ }^{\alpha}$ ). 
aufzeigen: auf die bisweilen in ihnen ausgetragenen Auseinandersetzungen der Priesterschaft eines Tempels mit den Trägern der politischen Macht.

Die śivaitischen Legenden Cidambarams haben ihren Ursprung in der großen südindischen Geschichtsepoche der ersten bedeutenden südindischen Reichsgründungen unter den Pallavas und den Cōlas (etwa 600 bis 1250 n. Chr.). Eingang in das $\mathrm{Cm}$ fanden jedoch nicht die volkstümlichen, tamilischen Legenden des 7. bis 9. Jahrhunderts n. Chr., in denen das Leben und die Taten der großen śivaitischen Nāyaṇārs in Cidambaram verherrlicht werden. Das $\mathrm{Cm}$ in seiner heute vorliegenden Form ${ }^{12}$ stammt aus dem 10. bis 12. Jahrhundert n. Chr. und stellt eine Zusammenfassung neuer Legenden dar. In ihnen setzt sich die Priesterschaft Cidambarams mit der volkstümlichen Tradition der frühen Legenden auseinander, bzw. gestaltet diese ihren eigenen Bedürfnissen entsprechend um. Im Mittelpunkt dieser neuen Legenden steht der berühmte ĀnandaTāndava-Tanz Sivas. Dieser Kult ist in Cidambaram nicht vor dem Beginn des 11. Jahrhunderts $\mathrm{n}$. Chr. belegbar. Von Cidambaram als Zentrum dieses Kultes breitete sich diese neue Tanzform Sivas vor allem in Südindien derart aus, daß seine Skulptur, die Ananda Coomaraswamy in seinem berühmten Artikel „The Dance of Shiva" beschrieben hat, heute zu den bekanntesten Symbolen des gesamten Hinduismus zählt.

Das $\mathrm{Cm}$ schildert in 26 Adhyāyas von je rund 50 Doppelversen die legendäre Geschichte Cidambarams. Nach den einleitenden Kapiteln $(\mathrm{I}-\mathrm{V})$ beginnt sie mit der Wanderung des Rși Vyäghrapāda nach Cidambaram, der dort ein Linga an der Sivagañgā, dem heiligen See Cidambarams, verehrte (VI-X). Es folgt dann nach einer langen Vorgeschichte die Schilderung der Ankunft Sivas in Cidambaram, wo jener zum ersten Mal seinen Ānanda-Tāṇḍava tanzt (XI-XVIII). Im letzten Drittel wird dann der Besuch des legendären Königs Hiranyavarman beschrieben, der aus Dankbarkeit für seine Heilung in der Sivagang $\bar{a}$ in Cidambaram 3000 Brahmanen ansiedelt, die Tempel Cidambarams erneuern läßt und dann ein großes Tempelfest stiftet.

Während wir in den beiden ersten Abschnitten nur auf legendäre Heilige stoßen, die sich einer historischen Einordnung entziehen ${ }^{13}$, tritt uns in Hiranyavarman eine Gestalt entgegen, deren legendäre Geschichte voller vager Anspielungen auf eine geschichtliche Situation ist. Vorwegnehmend sei hier auf die Uberreichung des Tigerbanners an Hiranyavarman hingewiesen. Das Tigerbanner stellt, allen interessierten Zeitgenossen des Verfassers unserer Legende wohl bekannt, das Wappensymbol der Cōlas dar. Die Hiranyavarman-Legende eignet sich wegen ihrer Transparenz historischer Ereignisse besonders gut, um an ihr einen bestimmten Aspekt der Entstehungsgeschichte und des Wandels einer südindischen Legende aufzuzeigen.

Im 25. Adhyāya wird ausführlich die großartige Erbauung Cidambarams durch den König Hiranyavarman geschildert, unter der in dem historischen Zusammenhang der Cōla-Dynastie nur eine Erneuerung verstanden werden kann. Harle kommt aufgrund umfangreicher Untersuchungen in Cidambaram zu dem wichtigen Ergebnis: „The vast majority of the older buildings which are still standing in the temple belong, without much doubt, to the time of Kulottunga I. (1070-1118) and his immediate successors, for whose building activity there is fortunately a good deal of evidence ${ }^{\text {14 }}$. Die älteste

12 Government Manuscript Library Madras, Ms. D 19347 und Ms. R 7632.

13 Patañjali, neben Vyäghrapāda und Hiranyavarman einer der drei großen Heiligen Cidambarams, wird von den orthodoxen Gelehrten Cidambarams selbstverständlich auch heute noch mit dem berühmten Grammatiker und dem nicht weniger bekannten Verfasser der YogaSütras identifiziert, die beide eben diesen Namen tragen. Die Gleichsetzung dieser großen Gelehrten ist ebenso unhaltbar (E. Frawwallner, Geschichte der indischen Philosophie [Salzburg 1953] Bd. I, S. 285) wie deren Identifizierung mit dem Patanjali des $\mathrm{Cm}$.

14 Harle (1963) S. 31. 
in situ erhaltene Inschrift Cidambarams stammt ebenfalls von Kulottunga $1 .{ }^{15}$ Von ihm und seinen Nachfolgern ist dann eine ununterbrochene Reihe von Inschriften vorhanden. Die kunstgeschichtliche und epigraphische Quellenlage in Cidambaram schließt daher eine grundlegende Umgestaltung Cidambarams, wie sie im $\mathrm{Cm}$ geschildert wird, und wie sie ja auch tatsächlich unter Kulottunga $I$. stattgefunden hat, nach Kulottuniga $I$. aus. Einzelne Angaben über von Hiranyavarman in Cidambaram errichtete Bauten wie Tortürme (gopura, XXV, 34) und Umwallungen (XXV, 33) der gesamten Tempelanlage schließen aus kunstgeschichtlichen Gründen eine Zeit vor etwa $1000 \mathrm{n}$. Chr. aus, da jene Bauten erst seit dem 11. Jahrhundert in größerem Maße in die Tempelarchitektur Südindiens einbezogen wurden ${ }^{16}$.

Betrachten wir das verbleibende 11. Jahrhundert als mögliche Entstehungszeit der Hiranyavarman-Legende, so scheidet die erste Hälfte des 11. Jahrhunderts wegen der zahlreichen Hinweise der Legende auf eine krisenhafte Situation im Reiche Hiranyavarmans $^{17}$ von vornherein aus: Diese Zeit stellt den Höhepunkt der Macht des CōlaReiches unter Räjaräja I. (985-1016) und Räjendra I. (1012-1044) dar, eine Zeit, in der die ersten monumentalen Reichstempel der Cōlas entstanden und in der Heere und Flotten dieser Dynastie bis nach Bengalen und Südostasien vordrangen. Die wiederholte Betonung politisch unruhiger Zeiten trifft im 11. Jahrhundert im Cōla-Reich einzig auf das Jahr 1070 zu. Nach dem Tode des Cōla-Königs Virarājendra zu Anfang des Jahres $1070^{18}$ vermochte der westliche Cālukya-Herrscher Vikramâditya VI. mit einem Heer bis tief in das Cōla-Reich vorzustoßen und seinen Günstling, den Cōḷa Adbiräjendra, in der Hauptstadt Ganigaikoṇdacōḷapuram zum neuen Cōla-König krönen zu lassen. Wenige Wochen später gelang es aber dem östlichen Cāḷkya Räjendra II. (später Kulottunga I.), auf bisher ungeklärte Weise den Cōla-Thron zu besteigen. Die Tatsache, daß es in beiden Fällen zu Interventionen fremder Mächte kam, ist in der Geschichte der Cōlas des 10. und 11. Jahrhunderts einzigartig. Der Kampf, der der Krönung Adbirājendras vorausging, kann jedoch kaum der Hintergrund für die HiranyavarmanLegende darstellen, da ja jener schon nach wenigen Wochen von der politischen Bühne verschwand. Um so deutlicher weist das Leben Räjendra II./Kulottunga I. eigenartige Parallelen zur Legende Hiranyavarmans auf.

Kulottuñga war Angehöriger der östlichen Cālukya-Dynastie, deren Reich sich an den südindischen Flüssen Godāvarī und Krșnāā erstreckte. Die Cālukyas waren sozusagen die Erbfeinde der Cōḷas, doch politische Umstände ergaben es, daß deren Seitenlinie, die östlichen Cālukyas, immer engere verwandtschaftliche Beziehungen mit den Cōlas einging, so daß Kulottunga I. blutsmäßig bereits mehr Cōla als Cālukya war. Nach dem Tode seines Vaters 1061 scheint Kulottunga als Räjendra II. Herrscher des östlichen Cālukya-Reiches gewesen zu sein. Etwa ein Jahr später wurde er jedoch von seinem Onkel Vijayâditya VII. vom Thron vertrieben. Uber sein weiteres Leben bis 1070, als er den Cōla-Thron bestieg, ist wenig Verläßliches bekannt.

Im folgenden seien nun einige der Gründe für die eigenartige Tatsache dargelegt, daß ein Herrscher des mächtigsten südindischen Reiches noch zu seinen Lebzeiten in die Reihe der

15 ARE 119/1888 bei Rangacharya (1919) Vol. I, S. 125 f. Siehe hierzu auch Balasubrabmanyan (1943) und (1944). $\quad{ }^{16}$ Harle (1963) S. 16 f., und Goetz (1962) S. 184.

17 Z.B. Cm XXIV, 53, wo Hiranyavarman von Vyäghranäda aufgefordert wird, die Herrschaft zu ergreifen: „Dein Vater ist vom Alter besiegt. Dein Reich ist obne Führung. Ergreife die Herrschaft!"

18 Sastri (1955) widmet der „Vorgeschichte ${ }^{\alpha}$ Kulottungas einen gesonderten Abschnitt (S. 285 bis 300 ), auf den ich mich hier vor allem stütze. 
legendären Gestalten einer berühmten Tempelstadt aufgenommen wurde. Diese Gründe lassen sich am anschaulichsten an Hand der Uberprüfung einiger Angaben der Hiranyavarman-Legende des $\mathrm{Cm}$ aufzeigen. Diese Legende beginnt mit einer ausführlichen Beschreibung der Ahnenreihe Hiranyavarmans, die bis zu den beiden Söhnen der Sonne, Manu und Yama, zurückverfolgt wird (XX, 4-XX, 37). Der sechste Angehörige dieser Sonnendynastie (süryavaṃśa) ist Hiranyavarman. Diese Zugehörigkeit zum sūryavamiśa wird in der Legende noch mehrfach ausdrücklich bestätigt (z. B. XXI, 51). Diese auffällige Hervorhebung der Abstammung Hiranyavarmans von der Sonne kann in Hinblick auf den König Kulottunga I. nur einen Zweck haben: Kulottunga ist als Cālukya Angehöriger der Monddynastie, während die Cōlas zur Sonnendynastie gerechnet werden. Um Kulottuniga vom „Makel ${ }^{\star}$ seiner Cālukya-Herkunft zu befreien, wird eine Ahnenreihe für Hiranyavarman / Kulottunga erfunden, die bis Manu, den Sohn der Sonne, zurückreicht, die Kulottunga nachträglich eine Grundvoraussetzung für seine Herrschaft über das Cōla-Reich erfüllen soll.

Als eine weitere wichtige Begebenheit schildert die Legende ausführlich die Adoption Hirañyavarmans durch Vyāghrapāda, den ersten Priester Cidambarams (XXII, 20-25). Hierzu ist es nun interessant zu hören, was Jayagondar, der Hofdichter Kulottungas, in seinem Hauptwerk, im Kalingattupparani, berichtet. Dort heißt es, daß Kulottunga als Cālukya-Prinz Räjendra II. von dem herrschenden Cōla-König Vìraräjendra $(1063-70)$ zu seinem Kronprinzen ernannt worden sei ${ }^{19}$, was mit einer Adoption gleichzusetzen wäre. Aus anderen Quellen wissen wir aber mit Sicherheit, daß von einer Adoption Kulottungas oder einer Ernennung zum Kronprinzen des Cōḷa-Reiches nicht die Rede sein kann. Nilakanta Sastri setzt sich ausführlich mit dieser Behauptung Jayagondars auseinander und kommt $\mathrm{zu}$ dem Schluß: „We have to assume, that the court poet of Kulottunga introduced this story to give validity to Kulottungas title to the Cōla throne. ${ }^{20}$ Diese Manipulationen finden eine Parallele in den Inschriften Kulottungas, in denen die Legitimität, bzw. sogar die Existenz seines Vorgängers, Adhiräjendra, der immerhin einige Wochen vor ihm nachweislich regierte, bestritten wird $^{21}$. Dieses bewußte Ubergehen seines Vorgängers zeigt, wie sehr sich Kulottuniga bemühte, den Eindruck zu erwecken, als sei er der direkte und legitime Nachfolger Virarājendras.

Die Hiranyavarman-Legende dürfte vermutlich einen weiteren Versuch enthalten, Kulottunga als Hiranyavarman zum direkten Angehörigen des Cōla-Hauses zu erheben. Der Vater Hiranyavarmans heißt Vikrama (XX, 34), trägt aber auch den Namen „bhaggirathī-püraparipanthin “ (XX, 35). Bhagirathī ist ein Name der Gañgā. Das Kompositum "bhägirrathi-püra-paripantbin ${ }^{*}$ dürfte in unserem Zusammenhang ${ }^{22}$ mit "einer der den Gañgä-Strom zu besiegen (bzw. zu umgehen) vermag " zu übersetzen sein. Eine sinnvolle Erklärung dieses Kompositums könnte in einer Anspielung auf den Eroberungszug der Heere des großen Cōla-Königs Rājendra I. bis zur Gañgā (um 1020-22) liegen, der das einzige historische Ereignis ist, das die Cōlas mit der Gañgā verbindet. Die Erinnerung an diesen Südindien tief beeindruckenden Eroberungszug

\footnotetext{
10 Sastri (1955) S. 293.

${ }^{20}$ Ebd., S. 297.

${ }^{21}$ Ebd., S. 294: (To) „cast doubt on the legitimacy of Adhirajendra's rule the poem and the inscriptions are in perfect agreement. ${ }^{\alpha}$

${ }_{22}$ paripanthin ist im $\mathrm{Cm}$ noch zweimal belegt. Einmal (XIII, 48) wird Siva als "nirghātaparipanthin ${ }^{\alpha}$ angerufen, was nur im Sinne von „Verbinderer der Zerstörung ${ }^{\alpha}$ aufgefaßt werden kann. Ein weiteres Mal wird Sivas Gesichtsfarbe bei seinem Tanz gepriesen, die die (Schönheit einer) „aufgeblühten, lieblichen Lotusblume besiegt ${ }^{*}$ (praphulla-ramaniyâbja-paripanthbimukhac= chavim, XVII, 47).
} 
wurde in dem Namen der neuen Hauptstadt Rājendras I., Gangaikoṇḍacōlapuram, festgehalten, für deren Weihe Rājendra heiliges Wasser der Gangā als „Beute ${ }^{\alpha}$ heimführte. Dieser Name rühmt den „Cōla Worten den Inhalt des Kompositums „bhāgīrathi-pūra-paripantbin " wieder. Ziehen wir des weiteren in Betracht, daß Räjendra I. in den Tiruvālàigädu-Inschriften ebenfalls „Vikrama $a^{\alpha}$ genannt wird ${ }^{23}$, also den Namen trägt, den Hiranyavarmans Vater im $\mathrm{Cm}$ (XX, 34) führt, und daß Kulottunga I. sich seinerseits Räjendra Cōladeva nennt ${ }^{24}$, so ist kaum daran zu zweifeln, daß im $\mathrm{Cm}$ der Eindruck einer ${ }_{\text {„Adoption }}{ }^{\alpha}$ Kulottungas an den Cōḷa-Hof unter seinem Großvater mütterlicherseits, Rājendra I., erweckt werden soll ${ }^{25}$. Diese Schilderung im $\mathrm{Cm}$ dürfte jedoch genauso wenig den Tatsachen entsprechen, wie der Bericht Jayagondars. Beide Beschreibungen dieser "Adoption" verraten aber dasselbe Anliegen ihrer Verfasser, nämlich Kulottungas Anrecht auf den Cōla-Thron durch eine fingierte Adoption in die Cōla-Familie nachzuweisen.

Durch die Aufstellung der Ahnenreihe bis Manu wurde Kulottunga in der Hiranyavarman-Legende seine Zugehörigkeit zur Sonnendynastie der Cōlas bestätigt. Die „Adoption" sollte seinen Anspruch, direkter Nachfolger der großen Cōlas zu sein, eine sakrale Weihe erteilen. Noch deutlicher wird die Absicht des Verfassers unserer Legende, Kulottungas Herrschaft über das Cōla-Reich zu sanktionieren, in der Beschreibung, wie Vyāghrapāda dem König Hiranyavarman ein Tigerbanner (vyāghradbvaja, XXV, 16) überreicht. Indem der Verfasser dieser Legende Vyäghrapäda, den ersten Priester des Reichtempels Cidambaram, Hiranyavarman das Tigerbanner darbringen läßt, das (wie schon erwähnt) zu dieser Zeit jedermann als das Wappen der Cōlas bekannt war, verläßt er das ungeschriebene Gesetz, nur allegorisch auf das politische Geschehen anzuspielen und es dadurch zu beeinflussen. Statt dessen benutzt er seine Macht, um durch eine von ihm geschaffene Legende offen Partei für seinen Herrscher zu ergreifen. Bisher war man geneigt, die Überreichung des Tigerbanners an Hiranyavarman als ein Beugen der Priester Cidambarams vor der neuen Macht der Cōlas nach ihrem Sieg über die Pallavas im späten 9. Jahrhundert n. Chr. zu deuten ${ }^{26}$. Sehen wir jedoch in Hiranyavarman den König Kulotturiga I., so muß diese Uberreichung des Tigerbanners im Zusammenhang mit den nicht $\mathrm{zu}$ übersehenden zahlreichen Hinweisen des $\mathrm{Cm}$ auf politisch höchst unruhige Zeiten gerade gegenteilig verstanden werden: Die Priester Cidambarams sanktionieren nachträglich die Herrschaft eines unrechtmäßig zur Macht gelangten Königs. Diese Deutung setzt voraus, daß seine Herrschaft Auseinandersetzungen um die Thronfolge vorausgegangen sind. Diese Voraussetzung triff in der gesamten Geschichte der Cōlas des 10. und 11. Jahrhunderts nur auf die Zeit Ku-

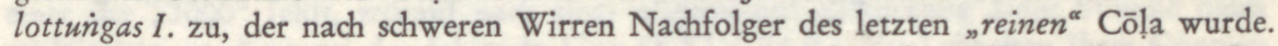
Kulottunga I. bedurfte somit wie kein anderer Herrscher dieser Dynastie einer Bestätigung seines Königtums.

In der ausführlichen Beschreibung der Wanderung Hiranyavarmans aus Nordindien nach Cidambaram (XX,50-XXI, 39), in deren Verlauf er zahlreiche Wallfahrtsorte

\footnotetext{
23 Sastri (1955) S. 206.

24 ARE 119/1888; Rangacharva, Bd. I, S. $125 \mathrm{f}$.

${ }^{25}$ Hierzu findet sich ebenfalls eine Parallele in Javagondars Kalingattupparani, wo es heißt, daß Rajendras I. Frau beim Anblick des neugeborenen Rajendra II. (später Kulottunga I.) erfreut ausgerufen hab, er sei fähig, ein Sohn der Sonnendynastie zu sein und jene dereinst zu beschützen (Aiyangar, Bd. I, S. 630).

26 Graefe, S. 14.
} 
besucht, dürfte ein weiterer Versuch liegen, Kulottungas Vergangenheit in einer Legende in ein wohlgefälliges Licht zu rücken. Eingangs wurde bereits erwähnt, daß Kulottuniga als Herrscher des östlichen Cālukya-Reiches von seinem Onkel Vijayâditya verdrängt worden ist, worauf er bis 1070 , als er sich des Cōla-Throns bemächtigte, ein recht unstetes Leben geführt zu haben scheint. In einer Inschrift verkündet er jedoch später einem seiner Söhne, daß er noch als Herrscher Verigis, der Hauptstadt seines Reiches, einen Welteroberungszug angetreten habe: „Liebes Kind! Früber habe ich die Herrschaft über das Verigi-Reich dem Prinzen Vijayâditya, meinem väterlichen Onkel, übertragen, (da) ich begierig war, eine Welteroberung durchzufübren. ${ }^{\alpha 2}$ Auch Jayagondar spricht im Kalingattupparani von einer Welteroberung, die Kulottunga unternommen haben soll ${ }^{28}$, die aber sonst durch nichts zu belegen ist. Die Vermutung liegt nahe, daß Hiranyavarmans Wallfahrt im $\mathrm{Cm}$ ebenso wie die „Welteroberung ${ }^{\circ}$ Kulottungas in seiner Inschrift und in seiner „vita“ des Jayagondar das gleiche Ziel verfolgen, nämlich Kulottungas unstetes und wohl auch ein wenig unrühmliches Leben zwischen 1062 und $1070^{29}$ einen schmeichelhafteren Ruf zu geben und es (dem Heiligen und dem König entsprechend) zur „Wallfabrt ${ }^{\alpha}$ bzw. zur „Welteroberung ${ }^{\alpha}$ umzudeuten ${ }^{30}$.

Die Liste der „politisch ${ }^{\text {“ }}$ bedingten Einzelheiten unserer Legende ließe sich noch erheblich erweitern. Aus ihr seien nur noch zwei Beispiele herausgegriffen, die das bisher Gesagte weiter bestätigen. In der Legende wird, sich fast stereotyp wiederholend, Hiranyavarmans rechtmäßiger Anspruch auf den väterlichen (Cōla-!)Thron (paitṛka simhāsana, XXV, 25) im eigenen (svīya) Palast betont. Eine derart häufige Hervorhebung ist kaum denkbar bei einem König, dessen Legitimität seinen Zeitgenossen keinen Anlaß zum Zweifel gegeben hat. Ähnlich auffällig wie verdächtig sind die wiederholten Aufforderungen an Hiranyavarman, seine rechtmäßige Herrschaft zu ergreifen (z. B. XXII, 5-6; XXIV, 53). Auch diese häufigen Ermahnungen blieben bei einem König, der rechtmäßig seinem Vater auf den Thron folgte, nur schwer erklärbar ${ }^{31}$. Anders hingegen bei Kulottunga I., dessen Cālukya-Herkunft seinen Zeitgenossen gut bekannt gewesen sein dürfte.

Ubereinstimmend lassen alle diese aufgeführten Belege einen (nach Berg) „optativen Charakter" erkennen, insofern sie alle den "rechtmäßigen" Anspruch Hiranyavarman / Kulottungas I. auf den Cōla-Thron betonen, - eben weil ein solcher Anspruch $\mathrm{n}$ i cht vorhanden war. Kein rechtmäßiger König der Cōlas hätte einer derartigen Bestätigung seines Königtums bedurft. Bei der Untersuchung derartiger Legenden und ähnlicher Quellen sollte es also der erste Schritt sein, deren „optativen Charakter" zu erkennen, da man sonst Gefahr läuft, ein Bild der Vergangenheit entstehen zu lassen,

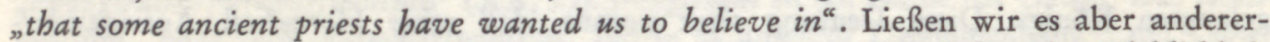
seits bei der Feststellung bewenden, daß hier ein Widerspruch zwischen der Wirklichkeit und dem Wunschbild vorliegt, so würden wir uns damit den weiteren Zugang zum

27 E. I., VI, S. 339; Vers 14.

28 Sastri (1955) S. 293.

29 1061 starb Kulottuñgas Vater Räjaräja Narendra. 1062 dürfte Vijayâditya die Herrschaft über Vengi übernommen haben.

so Nach Berg (1956) S. 174, hatte die Geschichte vom zwölfjährigen Aufenthalt des javanischen Königs Erlangga bei den Walderemiten (die sonst noch immer als erwiesen angesehen wird) ebenfalls den Zweck, „eine Theorie über den langjährigen Verbleib des Königs in der Wildnis, bevor er König wurde, in den Umlauf zu bringen ${ }^{\text {. }}$.

31 Auch hier drängt sich wie in der Anm. 30 der Vergleich mit der javanischen ErlanggaLegende auf, in welcher der (unrechtmäßig) zur Herrschaft gelangte König von den Priestern ersucht worden sein soll, die Herrschaft über den herrenlosen Staat zu übernehmen (Berg [1956] S. 172 ff.). 
Verständnis dieser Legenden verbauen. Denn ihr Wesen liegt ja gerade darin, ein Wunschbild zur neuen „legendären Wirklichkeit “ werden zu lassen.

Wer aber waren nun die Priester, die Kulottuniga I. schon zu seinen Lebzeiten in den Kreis ihrer Heiligen aufgenommen haben und worin bestand die Ursache, daß sie in einem derartigen $\mathrm{Maß}$ auf die Bedürfnisse eines Königs eingingen?

In die Legende Hiranyavarmans ist geschickt die Geschichte der „3000 Brahmanen ${ }^{\text {“ }}$ eingeflochten (XXIV, 58-86), die der König Hiranyavarman auf Anraten des Priesters Vyāghrapāda aus dem Antarvedī-Land nach Cidambaram holt. Wie aber aus einer von Hultzsch $^{32}$ edierten Inschrift aus der Entstehungszeit unserer Legende hervorgeht, kann in dem südindischen Kontext der Legende mit Antarvedī nicht das nordindische Zweistromland zwischen der Gañgā und der Yamunā gemeint sein, sondern nur dasjenige zwischen den südindischen Flüssen Godāvarī und Krṣnnā. Eben diese Gegend ist aber das Kerngebiet des heimatlichen Reiches Kulottungas. Die Priester, die Hiranyavarman/ Kulottunga in Cidambaram ansiedelte, müssen demnach Priester aus seinem eigenen Lande gewesen sein.

Diese Schilderung der Ansiedlung mag durchaus auf einer historischen Begebenheit beruhen ${ }^{33}$, da für einen Ursupator des Cōla-Throns die Anwesenheit ihm ergebener Priester in Cidambaram, dem sakralen Zentrum des Reiches, von großer Bedeutung gewesen sein muß. In diesem Zusammenhang ist das Verhalten des Jațāvarman Sundara Pāndyas (1251-68) aufschlußreich, dem mächtigsten Herrscher der "späten " Pändyas, die im 13. Jahrhundert endgültig die Cōla-Hegemonie über Südindien beendeten. Jațāvarman vollbrachte in Cidambaram ähnliche Taten wie vor ihm die Cōla-Könige. So ließ er sich z. B. gegen Gold aufwiegen, um damit die Tanzhalle Sivas in Cidambaram zu vergolden ${ }^{34}$. Eine Inschrift Cidambarams ${ }^{35}$ berichtet von einer „agrabāra ${ }^{\text {c }}$ ([königlichen] Landschenkung für Brabmanen) - Dorfgründung für 108 Brahmanen in der Nähe Cidambarams. Diese Ansiedlung der 108 Brahmanen in der Krönungsstadt der von ihm besiegten Cōlas dürfte ähnlich einleuchtende Gründe haben, wie die in der Hiranyavarman-Legende berichtete Ansiedlung der "3000" durch Hiranyavarman/ Kulottunga $I$.

Der Verfasser unserer Legende dürfte unter diesen Priestern zu suchen sein. Doch sein Werk beschränkt sich keineswegs darauf, die Herrschaft seines Königs über das CōlaReich zu sanktionieren. Er bescheinigt zusätzlich sich und den anderen neuen Priestern aus dem Cāḷkya-Reich eine legendäre Herkunft, indem er die ihrige mit derjenigen des legendären Königs Hiraṇyavarman verknüpft. Ferner bestätigt er ihnen, daß Siva einer der Ihren sei, indem er die Geschichte erzählt, daß bei ihrer Ankunft in Cidambaram einer der „3000“ fehlt, worauf nach einer allgemeinen Bestürzung Sivas Stimme dann verkündete, er, Śiva, sei der fehlende Eine (XXIV, 74 ff.). Zu Lebzeiten ihres Gönners mag es den alteingesessenen Priestern gegenüber ein Vorteil gewesen sein, Günstlinge eines mächtigen Herrschers zu sein. Sobald aber nach dessen Tode das Andenken an ihn verblaßte, mußte diese Beziehung in einen erheblichen Nachteil gegenüber den Alteingesessenen umschlagen. Denn jene vermochten es, über ihren ersten Priester, Vyäghra-

32 E. I., IV, S. 36; Sastri (1955) S. 320.

${ }^{33}$ Die Ansiedlung der ${ } 3000^{\circ}$ ist inschriftlich nicht belegbar. Sicher ist aber, daß sie vor der Entstehungszeit unserer Legende in der Literatur $\mathrm{n} \mathrm{i} \mathrm{cht} \mathrm{nachweisbar} \mathrm{sind,} \mathrm{während} \mathrm{sie} \mathrm{nach}$ dem 12. Jahrhundert zum festen Bestand der Heiligkeit Cidambarms zählen (z. B. in Umäpatis Kuñcitânghristaya, um 1300).

${ }^{34}$ Sastri (1966) S. 215; S. I. I., No. 620.

35 ARE 277/1913; Rangacharya, Vol. I, S. 134. 
pāda, ihre Geschichte bis zur Teilnahme an dem mythischen Urgeschehen Cidambarams, Sivas Ānanda-Tāṇḍava zurückzuverfolgen, während demgegenüber die „3000“ mit dem „Makel ${ }^{\alpha}$ behaftet waren, erst später von einem König in Cidambaram angesiedelt worden zu sein.

Eine textkritische Untersuchung zeigt nun in aller Deutlichkeit den Versuch der „3000“, ihre legendäre Geschichte nachträglich bis zu dem Besuch Sivas in Cidambaram „zurückzwverlängern ${ }^{\alpha}$. In einer Reihe von Interpolationen wird geschickt nachgewiesen, daß die "A nsiedlung “ durch den König in Wirklichkeit nur eine "R ü cksied$1 \mathrm{u}$ n " " gewesen sei. Hierzu wird unter anderem Adbyäya XIX. erfunden, in dem geschildert wird, wie Vyăghrapāda die 3000 Munis in Vertretung für die in Cidambaram weilenden Götter zu einem Opfer Brahmās an die Gangā geschickt hat, was heißt, daß die 3000 Priester schon v or Hiranyavarman in Cidambaram ansässig waren. In weiteren Interpolationen vor und nach der Beschreibung des Tanzes Śivas (XVII, 9-89) wird betont, daß die „3000“ zunächst gemeinsam mit Vyāghrapāda auf Siva warteten (XVII, 4-8) und daß sie ihn dann „sehr klar ${ }^{*}$ gesehen haben (XVIII, 1-4). Aus dem ursprünglichen Text geht dagegen eindeutig hervor, daß nur die Götter, Vyāghrapāda und Patañjali Zeugen des ersten Tanzes Sivas waren.

Gleichzeitig mit diesen Interpolationen werden weitere, höchst aufschlußreiche Anderungen am Text der Legende vorgenommen. Immer dann, wenn in der ursprünglichen Legende der König Hiranyavarman den 3000 Priestern eine Anordnung erteilt (z. B. $\mathrm{XXV}, 52 \mathrm{ff}$.$) , wird, mehr oder weniger geschickt, nachträglich dieser Aufforderung eine$ Rede Vyäghrapādas „vorgespannt" (z. B. XXV, 49-51), in der dieser dem König genau das aufträgt, was jener einige Zeilen später dann den 3000 Munis anordnen wird. So entsteht der Eindruck, daß alles letztlich nur nach dem Willen Vyāghrapādas, dem ersten Priester Cidambarams, geschieht.

All diese Änderungen am ursprünglichen Text der Legende sind mit großer Sicherheit nach Ablauf eines Jahrzehnts nach dem Tod Kulottunigas I. vorgenommen worden ${ }^{36}$. Die Suche nach der Ursache für die erstaunliche Geschwindigkeit, in der die Änderungen an der Legende Hiranyavarmans vorgenommen wurden, führt wieder unmittelbar zu dem Aspekt dieser Literaturgattung zurück, der hier besonders interessiert.

Ein Māhātmya spielte offenbar für die Formulierung der Ansprüche und Wünsche der Priester eines Tempels und bei der Widerlegung fremder Ansprüche eine entscheidende Rolle. Die Bedeutung eines Mābātmyas wächst insofern, als wir ganz den Eindruck haben dürfen, daß Formulierungen, die in der geschilderten Weise in das offizielle Mābātmya eines Tempels aufgenommen werden, über kurz oder lang zum dogmatischen Bestandteil der Lehre dieser Tempelstadt werden, - falls ihnen nicht 'auf derselben Ebene der Mābātmyas widersprochen wird. Nur diese Tatsache vermag meines Erachtens die Schnelligkeit zu erklären, mit der in der Hiranyavarman-Legende der Abschnitt über die Herkunft der ${ }^{3} 3000$ “ korrigiert wurde oder die Gründlichkeit, mit der Anordnungen des Königs nachträglich zu Wünschen der Priester umgedeutet wurden.

Eine weitere Aufgabe dürfte einem Māhātmya in der Auseinandersetzung zwischen der Priesterschaft eines Tempels und den Trägern der politischen Macht zufallen. Die

${ }^{36}$ In der Indra-Legende des Cm (VII, 1-47), die ebenfalls zu den Interpolationen der Hiranyavarman-Schicht des $\mathrm{Cm}$ zählt, wird die große Skulptur Visnus in Cidambaram beschrieben, wie sie seit Mänikkaväśagar (frühes 9. Jh. n. Chr.) belegt ist. Diese Skulptur ließ der fanatische Sivait Kulottunga II. (1133-1150) aus Cidambaram gewaltsam entfernen. Seit Kulottunga II. (bis in die Zeit des Vijayanagara-Königs Acyutadeva-Rāya [1529-1542], der diese Skulptur wieder feierlich einweihen ließ) wäre eine Einbeziehung der Indra-Legende, in der Vișnu - in der Form der von Kulottuñga II. zerstörten Skulptur - eine beträchtliche Rolle spielt, in das $\mathrm{Cm}$ nicht denkbar. - 1122 ist das Datum der letzten Inschrift Kulottungas $I$. 
Hiranyavarman-Legende des $\mathrm{Cm}$ läßt deutlich erkennen, wie eng die Bindungen der Priesterschaft eines Tempels an einen König zu sein vermögen, wenn beide Seiten gemeinsame Interessen verbinden (z. B. die gegenseitige „Bescheinigung ${ }^{\alpha}$ einer legendären Herkunft). Die gründliche Überarbeitung läßt aber auch vermuten, wie gefährlich es werden kann, wenn diese Beziehungen in eine Abhängigkeit der Priester von der herrschenden Dynastie umschlagen. Dieser negative Aspekt einer engen Bindung scheint dann in den Vordergrund zu treten, wenn der König, dem die Priester aufgrund ihrer engen Beziehungen zu ihm einen beträchtlichen Einfluß auf das kultische Geschehen ihres Tempels gewährt haben, zu einer legendären Gestalt wird und Aufnahme in das Mābātmya eines Tempels findet. Die Verherrlichung derartiger Taten eines Königs in einer Legende könnte Vorbild oder Anlaß für Aktionen eines seiner Nachfolger werden, die möglicherweise dann nicht mehr im Sinne der Priester liegen.

In dieser Gefahr glaube ich, die eigentliche Ursache für die systematische Umarbeitung der Hiranyavarman-Legende sehen zu müssen. Die Interpolationen dürfen zwar auch dem Wunsch entsprungen sein, einen legendären König zum willfährigen Ausführenden der Wünsche der Priester zu erklären. Die überraschende Geschwindigkeit aber, mit der diese Änderungen an der Legende vorgenommen wurden, dürfte wesentlich darauf zurückzuführen sein, daß von vornherein verhindert werden sollte, einen König, der der Priesterschaft eines Tempels Anordnungen erteilt, zur "legendären Wirklichkeit ${ }^{\star}$ werden zu lassen.

Anfangs wurde angedeutet, daß Bergs Methode einer "funktionalen Interpretation " javanischer historischer Texte nur mit einer Einschränkung auf gewisse indische Verhältnisse übertragbar ist. Diese Einschränkung beruht auf der unterschiedlichen Bedeutung der Wort- und Schriftmagie in Indien und in Java. In der Zeit, aus der uns historische oder quasi-historische Quellen überliefert sind, dürfen wir in Indien nicht in dem Maße mit einem Glauben an die Macht der Magie rechnen, wie wir ihn im mittelalterlichen Java voraussetzen müssen. In Java dürfte in der Tat den "Priestern die Aufgabe zugefallen sein", dem "Königtum das sakrale Fundament zu geben. ${ }^{\text {" }}{ }^{37}$ Der Glaube an die Wirksamkeit priesterlicher Magie war in Java imstande, dieses „Fundament ${ }^{\text {“ }}$ zu erschaffen. Ein ähnlich wirksamer Glaube an die Macht der Magie dürfte z. B. in der Zeit der Brāhmanas in Indien durchaus vorhanden gewesen sein. Später, in der Zeit der mittelalterlichen Staatenbildungen, mag er auch noch in den altertümlichen Opfern wie im Aśvamedha oder im Krönungsritual weitergelebt haben, doch wurde er durch die in der Zwischenzeit entstandene Pluralität der philosophischen und politischen Systeme Indiens in Frage gestellt. Dies mußte zur Folge haben, daß Magie nicht mehr als geeignetes Mittel angesehen werden konnte, einem fragwürdigen Anspruch auf das König-

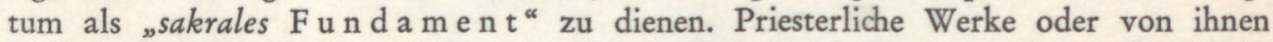
beeinflußte Inschriften vermochten in diesem Sinne in Indien nur, eine nachträgliche sakrale Sa nktionierung einer neuen Situation zu bewirken, bzw. die Wunschbilder zu einer "legendären Wirklichkeit ${ }^{*}$ werden zu lassen.

Diese Gegenüberstellung ist notwendig, um auf die unterschiedliche Wirkung politischreligiöser Texte hinzuweisen. Die unmittelbar „wirkende ${ }^{\star}$ magische Manipulation mag ihren historischen Kontext zunächst stärker beeinflußt haben, als „politisch ${ }^{\text {“ bedingte }}$ Legenden, die länger benötigt haben dürften, um das Medium ihrer Wirksamkeit, die volkstümliche Uberlieferung, zu erreichen. Doch durch ihr Eindringen in die traditionsfreudige Welt der Legenden und Mythen haben letztere ihren jeweiligen historischen Kontext dauerhafter beeinflußt.

${ }^{37} \operatorname{Berg}(1956)$ S. 173. 


\section{Abkürzungs- und Literaturverzeichnis (Auswabl)}

Aiyangar, S. K. (1941): Ancient India and South Indian History and Culture (Poona) 2 Vols. [= Poona Oriental Series No. 74].

ARE $=$ Annual Reports on Epigraphy (Madras).

Balasubrahmanyan, S. R. (1943) und (1944): The Oldest Chidambaram Inscriptions, in: Journal of the Annamalai University, Vol XII, S. 106-118, und Vol XIII, S. 55-91.

Berg, C. C. (1956) und (1957): Javanische Geschichtsschreibung, in: Saeculum 7 (1956) S. 168 bis 181 , und 8 (1957) S. 249-266.

- (1961): Javanese Historiography - A Synopsis of its Evolution, in: D. G. E. Hall (ed.), Historians of South-East Asia (Oxford University Press, London) S. 13-23.

- (1965): The Javanese Picture of the Past, in: Soedjatmoko (ed.), An Introduction to Indonesian Historiography (Cornell University Press, New York) S. 87-118.

$\mathrm{C} m=$ Cidambaramāhātmya, MSS., Government Manuscript Library, Madras, Nos. D 19347 und R 7632. Zitiert nach D 19347.

$\mathrm{EI}=$ Epigraphia Indica.

Goetz, H. (1962): 5000 Jahre Indische Kunst (4. Aufl. Baden-Baden).

Graefe, W. (1960): Legends as Mile-Stones in the History of Tamil Literature, in: Prof. P. K. Gode Commemoration Volume, Vol II (Poona) S. 129-146; im Sonderdruck: S. 1-18.

Harle, J. C. (1963): Temple Gateways in South India. The Architecture and Iconography of the Cidambaram Gopuras (Oxford).

Kulke, H. (1968/69): Cidambaramāhātmya. Eine Untersuchung der religionsgeschichtlichen und historischen Hintergründe für die Entstehung der Tradition einer südindischen Tempelstadt. Erscheint 1968/69 als Band 3 in der von Prof. U. Schneider herausgegebenen Reihe „Freiburger Beiträge zur Indologie " (Harrassowitz, Wiesbaden).

PW = Petersburger Wörterbuch.

Ramachandran, T.N. (1948): Siva Temple of Chidambaram, in: Journal of the Andhra Historical Research Society, Vol XIX, S. 85-87.

Rangacharya, V. (1919): Topographical List of the Inscriptions of the Madras Presidency, 3 Bde. (Madras).

Sastri, K. A. N. (1932): Studies in Chola History and Administration [= Madras University Historical Series, No. 7 (Madras)].

- (1955): The Cōlas. 2nd ed. [= Madras University Historical Series No. 9 (Madras)].

- (1966): A History of South India (Oxford University Press, Madras [3rd. ed]).

Sewell, R. (1932): The Historical Inscriptions of Southern India, Collected till 1923 (Madras). SII $=$ South Indian Inscriptions.

Varma, V. P. (1959): Studies in Hindu Political Thought and its Metaphysical Foundations (2nd ed. Delhi).

Winternitz, M. (1909-1920): Geschichte der indischen Literatur, 3 Bde. (Leipzig). 